\title{
PRÁTICAS INTEGRATIVAS E COMPLEMENTARES EM SAÚDE JUNTO A PROFISSIONAIS DA ATENÇÃO PRIMÁRIA
}

\section{Integrative and complementary practices in health with primary care professionals \\ Practicas integradas y complementarias de salud con profesionales de la atención primaria}

\author{
Isaac Newton Machado Bezerra iD \\ Universidade Federal do Rio Grande do Norte - UFRN - Natal (RN) - Brasil
}

\author{
Vinicius Costa Maia Monteiro iD \\ Instituto Técnico do Brasil - ITEC - Açu - (RN) - Brasil
}

Jânio Luiz do Nascimento (iD

Universidade Federal do Rio Grande do Norte - UFRN - Natal (RN) - Brasil

Larissa Oliveira Lima Macedo (DD

Universidade Federal do Rio Grande do Norte - UFRN - Natal (RN) - Brasil

Zacarias Ramalho Silvério (iD

Faculdade Estácio - FATERN - Natal (RN) - Brasil

Aurélia de Oliveira Bento iD

Secretaria de Saúde de Santo Antônio - Santo Antônio - Natal (RN) - Brasil

Francisco Canindé dos Santos Silva (iD

Universidade Potiguar - UnP - Mossoró (RN) - Brasil

Jônia Cybele Santos Lima iD

Universidade Federal do Rio Grande do Norte - UFRN - Natal (RN) - Brasil

\section{RESUMO}

Objetivo: Descrever as ações de Práticas Integrativas e Complementares em Saúde realizadas com trabalhadores da Atenção Primária. Síntese dos dados: Trata-se de um relato de experiência, realizado em 2017 e 2018, por estudantes dos cursos do Centro de Ciências da Saúde (CCS) da Universidade Federal do Rio Grande do Norte (UFRN), em parceria com os profissionais de uma Unidade de Saúde da Família (USF), em um município do Rio Grande do Norte, Brasil. Realizaram-se encontros na unidade de saúde voltados aos cuidados dos profissionais, utilizando-se Práticas Integrativas e Complementares em Saúde (PICS), como reike, shiatsu e corredor do cuidado, com atividades conduzidas por profissionais capacitados e com momentos direcionados à escuta dos profissionais e dinâmicas em grupo. Conclusão: As ações executadas se mostraram de extrema relevância, trazendo aos envolvidos o despertar para a importância do autocuidado. Foram momentos ricos de troca de saber e envolvimento dos profissionais e estudantes, que puderam compreender a dinâmica dentro dos serviços de saúde e buscaram juntos alternativas para o desenvolvimento da promoção do cuidado dos profissionais de saúde.

Descritores: Atenção Primária a Saúde; Saúde do Trabalhador; Promoção da Saúde.

\section{ABSTRACT}

Objective: To describe the actions of Integrative and Complementary Practices in Health performed with primary care workers. Data synthesis: It is an experience report, carried out in 2017 and 2018, by students of the courses of the Centro de Ciências da Saúde (CCS) of the Federal University of Rio Grande do Norte (UFRN), in partnership with the professionals of a Unidade de Saúde da Familia (USF), in a municipality of Rio Grande do Norte, Brazil. Meetings were held at the health unit dedicated to the care of professionals, using Integrative and Complementary Practices in Health (PICS), such as reiki, shiatsu, and care runner, with activities conducted by trained professionals and with moments directed to listening to professionals and group dynamics.

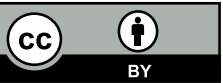


Conclusion: The actions performed were of extreme relevance, bringing to the participants the awakening to the importance of self-care. They were rich moments of exchange of knowledge and involvement of professionals and students, who could understand the dynamics within health services and sought together alternatives for the development of the promotion of care of health professionals.

Descriptors: Primary Health Care; Occupational Health; Health Promotion.

\section{RESUMEN}

Objetivo: Describir las acciones de Prácticas Integradas y Complementarias de Salud realizadas con trabajadores de la Atención Primaria. Síntesis de los datos: Se trata de un relato de experiencia realizado en 2017 y 2018 por estudiantes de los cursos del Centro de Ciencias de la Salud (CCS) de la Universidad Federal de Río Grande do Norte (UFRN) en colaboración con los profesionales de una Unidad de Salud de la Familia (USF) de un municipio de Rio Grande de Norte, Brasil. Se realizaron encuentros en la unidad de salud orientados para los cuidados de los profesionales utilizándose Prácticas Integradas y Complementarias de Salud (PICS) como el reike, el shiatsu y el pasillo del cuidado con actividades realizadas por profesionales capacitados y momentos indicados para la escucha de los profesionales y las dinámicas de grupo. Conclusión: Las acciones ejecutadas han sido de extrema relevancia conduciendo los involucrados para el despertar sobre la importancia del auto cuidado. Han sido momentos llenos de intercambio de saberes y envolvimiento de parte de los profesionales y estudiantes que han podido comprender la dinámica dentro de los servicios de salud y han buscado juntos las alternativas para el desarrollo de la promoción del cuidado de los profesionales sanitarios.

Descriptores: Atención Primaria de Salud; Salud Laboral; Promoción de la Salud.

\section{INTRODUÇÃO}

O direito à saúde está presente na Constituição Federal de 1988, que estabelece que esse direito seja inalienável e que cabe ao Estado prover aos seus cidadãos o acesso à saúde através de políticas sociais e econômicas ${ }^{(1)}$.

A partir da Consolidação das Leis Trabalhistas (CLT), em 1943, a obrigatoriedade da composição dos serviços de medicina do trabalho nas empresas de grande porte trouxe um panorama de progresso para as ações de saúde do trabalhador, embora tenha caminhado a passos lentos. A criação da Comissão Interna de Prevenção de Acidentes (CIPA) é obrigatória, porém seu surgimento foi de forma desordenada, sendo formados apenas por representações do patronato e, assim, mesmo os trabalhadores sofriam pressões da força de ameaças dos seus empregadores ${ }^{(2)}$.

Através dos anos, as políticas que visam garantir o direito à saúde dos trabalhadores e das trabalhadoras vêm sofrendo mudanças e sendo aprimoradas, a fim de promover o acesso a todos. Com a consolidação do Sistema Único de Saúde (SUS), a saúde passa a abranger todas as classes sociais, e é nesse novo cenário que, em dezembro de 2009, através da Portaria GM/MS n. ${ }^{\circ}$ 3252, cria-se a Vigilância em Saúde do Trabalhador (VISAT), definida como componente do Sistema Nacional de Vigilância em Saúde,em 2013, essa portaria é revogada pela Portaria n. ${ }^{0} 1.378$, de 9 de julho de $2013^{(3-5)}$.

A incorporação da lógica da Saúde Pública, de prevenção de riscos e de promoção da saúde com a participação dos trabalhadores, em uma perspectiva coletiva, constituindo o que se denomina como Saúde do trabalhador, efetivou-se no país a partir da criação do SUS, em 1988. Progressivamente, foram criados órgãos importantes para consolidação do direito a saúde do trabalhador em parceria com o SUS, como o Centro de Estudos em Saúde do Trabalhador e Ecologia Humana/Fiocruz, Cesteh/Fiocruz, o Instituto Nacional de Saúde no Trabalho (INST), entre outros, para articulação das lutas na garantia da saúde do trabalhador ${ }^{(2)}$.

A Política Nacional de Promoção da Saúde (PNPS) surge como um esforço coletivo de todas as áreas do Ministério da Saúde na busca da superação de um contexto histórico complexo da produção do cuidado, visando abarcar todas as lacunas do cuidado, compreendendo também a inserção da saúde dos trabalhadores do SUS(6).

A literatura aponta para um adoecimento acelerado dos profissionais que atuam na Atenção Primária de Saúde, com uma prevalência das doenças ligadas ao processo de trabalho, como transtornos de ansiedade, depressão e a síndrome de Burnout (SB) ${ }^{(7)}$.

A incidência de SB é alta entre os trabalhadores da saúde e, nos casos mais graves, pode causar depressão e suicídio, o que mostra a importância de se prevenir e tratar os $\operatorname{casos}^{(8)}$. Não é raro encontrar pesquisas que mostram o adoecimento ou queixas dos profissionais que compõem as equipes de Saúde da Família (ESF) que, apesar de relatarem ausência de enfermidades, queixavam-se de dores e de episódios depressivos ${ }^{(9)}$. 
Somente em 2011, a Mesa Nacional de Negociação Permanente do SUS - MNNP-SUS, por meio do Protocolo n. ${ }^{\circ}$ 008/ 2011, instituiu as Diretrizes da Política Nacional de Promoção da Saúde do Trabalhador do SUS. Esse documento empodera o trabalhador e traz as melhorias necessárias das condições de trabalho, assegurando o cumprimento de requisitos presentes na legislação vigente no país, fortalecendo a implementação de programas de proteção à saúde dos trabalhadores ${ }^{(10)}$.

Discutir a saúde do trabalhador da saúde é de extrema importância, pois os profisssionais de saúde não se percebem nesse cuidado. O incentivo para a promoção de ambientes de trabalho saudáveis, com ênfase na redução dos riscos de acidentes de trabalho e de doenças relacionadas ao trabalho, é importante. Um estudo reforça que o profissional que exerce o cuidado para com o outro, por diversas vezes, não se reconhece como alguém que também necessita de cuidado e, dessa forma, acaba adoecendo(11).

Ressalta-se, então, a relevância do presente estudo, pois possibilita alertar gestores e profissionais de saúde sobre a evidente necessidade de envidar esforços para que as ações para a saúde do trabalhador estejam pautadas na promoção da saúde e na prevenção de agravos.

Assim, diante desse contexto, o estudo tem por objetivo descrever as ações de Práticas Integrativas e Complementares em Saúde realizadas com trabalhadores da Atenção Primária.

\section{SÍNTESE DOS DADOS}

Trata-se de um relato de experiência, realizado por estudantes de cursos do Centro de Ciências da Saúde (CCS) da Universidade Federal do Rio Grande do Norte (UFRN), junto com profissionais da Unidade de Saúde da Família (USF) Justiniano Homem de Siqueira, situado no bairro Potengi - conjunto Panatis, Natal, Rio Grande do Norte, Brasil. Essa experiência fez parte do Programa Curricular de Orientação Tutorial para o Trabalho Integrado em Saúde (POTI) nos anos 2017 e 2018.

O componente curricular POTI é ofertado pela UFRN em todos os semestres, sendo obrigatório para os cursos de Medicina, Enfermagem e Odontologia, e optativo para os demais cursos da área da saúde. Como avaliação final, é solicitado que os alunos realizem uma atividade junto aos profissionais da unidade. Essa atividade é pactuada no início do semestre letivo, em que é realizada a definição do tema, em comum acordo entre alunos e profissionais da unidade, necessitando estar ligada a algum programa/projeto existente e em execução pelos profissionais. No decorrer do semestre, os alunos vivenciam o espaço de trabalho na Unidade Básica (UBS) da Estratégia Saúde da Família, observando o ambiente. Então, junto com os profissionais e a gestão, decidem qual a temática e qual a melhor forma de interagir com os profissionais. Após a definição, a atividade é traçada e apresentada aos profissionais da unidade para que seja aprovada por todos.

Tendo definido a saúde do trabalhador como assunto a ser abordado pela turma de 2017.1, os alunos elaboraram o cronograma de ações. Em seguida, foi realizada uma reunião com a gerência da UBS para aprovação e posterior início das atividades definidas.

A primeira ação realizada na unidade foi junto ao grupo de saúde do trabalhador (ST) da unidade Panatis, projeto escolhido pelos profissionais apoiadores para contar com a ajuda dos estudantes.

Essa atividade contou com dois momentos distintos, em dias diferentes, e envolvendo a saúde do trabalhador do SUS. No primeiro encontro foi realizada uma palestra sobre o adoecimento dos profissionais de saúde pela sobrecarga de trabalho e pelo esquecimento de que ele também necessita de cuidados. Diversos fatores são apontados para o adoecimento dos profissionais, como a falta de autonomia para decidir sobre onde trabalhar, conflitos no ambiente de trabalho, a violência e falta de estrutura adequada ${ }^{(12,13)}$. Também há relatos sobre a deficiência da descrição das atribuições dos profissionais que compõem a $\operatorname{ESF}^{(14)}$.

Esse momento foi conduzido por uma enfermeira especialista em saúde do trabalhador, apresentando números relativos ao adoecimento desses profissionais e o desenvolvimento da Síndrome de Burnout.

A Síndrome de Bournout traduz um esgotamento físico e mental, e está diretamente ligada a sentimentos de desagrado com as funções desempenhadas, principalmente quando essas estão relacionadas ao não alcance das metas e carga horária semanal superior a 30 horas $^{(15,16)}$. Estudos já evidenciam que profissionais mais jovens apresentam um maior risco de desenvolver a Síndrome de Bournout, e necessitam de um olhar mais apurado nesse sentido ${ }^{(17-19)}$.

A reação dos profissionais à palestra foi extremamente positiva, era notório que o momento havia alcançado o seu objetivo, qual seja: despertar neles o interesse em olhar um pouco mais para si e para seus colegas, percebendo a necessidade do autocuidado e do olhar para o outro. Para os alunos promotores da experiência também foi 
importante, pois representou um momento de reflexão de como será a atividade profissional futuramente e o que se poderá fazer para minimizar tal problemática.

A segunda parte da ação também contou com a execução de PICS. Dessa vez, realizou-se o shiatsu, técnica oriental de massagens ${ }^{(20)}$, com demonstração nos profissionais da unidade feita por uma psicóloga especialista na técnica. O reiki, técnica oriental baseada na transferência da energia universal, o ki ${ }^{(20)}$, também foi utilizada, sendo realizada por uma pedagoga reikiana.

As PIC são estratégias de cuidado que estimulam a percepção ampliada do processo saúde-doença e que buscam a interação entre natureza, homem e sociedade, e já vem sendo discutida e estudada sua inserção nos diversos níveis de complexidade dos sistemas de saúde ${ }^{(21-23)}$.

A utilização das PIC não busca a substituição ao modelo vigente, que utiliza a mais moderna tecnologia, tantos nos serviços privados quando no SUS, mas, sim, ser complementar a ele e apresentar-se como um modelo mais humano, em que a interação entre cuidador e paciente seja mais forte e presente ${ }^{(24)}$.

Todos os momentos foram orientados por especialistas da área em questão, levando em consideração os riscos envolvidos na realização de alguma prática por uma pessoa despreparada.

Em 2018.2, em parceria com os alunos matriculados na disciplina de POTI do referido semestre, criou-se um grupo de escuta e acolhimento para os profissionais da UBS. Como no ano anterior, dois momentos foram pactuados.

Como ponto de partida para a primeira reunião, colocou-se, dentro da USF Panatis, uma caixa voltada à escuta, na qual os profissionais deveriam colocar, de forma anônima ou não, algo que o entristecia com relação à interação com seus colegas e à execução de seu trabalho.

Os papéis foram retirados e lidos no encontro realizado com os profissionais que integram as esquipes da unidade. Algumas questões foram mais prevalentes, como a chateação por profissionais chegarem de mau-humor e deixarem isso passar para os demais e a irritação quando um profissional não atende um usuário de forma cortês, deixando de dar uma informação, por exemplo.

Esse momento foi importante para que os profissionais da unidade compreendessem quais atitudes, mesmo que minimamente, causavam desconforto nos colegas. Alguns dos presentes se manifestaram sobre as inquietações lidas para concordar e também para colocar como a situação poderia ser resolvida.

O segundo momento foi direcionado à diversão, focando na realização de diversas dinâmicas, sempre priorizando a interação entre os profissionais. Em uma das dinâmicas, solicitou-se que fizessem duplas para realizar uma dança que seria ensinada pelos alunos. Este representou o momento de maior descontração, em que todos participaram e no qual ficou nítida a satisfação presente.

O protagonismo e o engajamento dos profissionais da unidade foram de extrema importância para a efetivação dos momentos realizados. Devido ao desejo demonstrado por eles, os espaços foram ricos e cheios de significados e não limitados apenas àqueles momentos, mas continuam a existir mesmo sem a presença dos alunos.

A realização de vivências dentro do Sistema Único de Saúde (SUS) é primordial para o despertar profissional, podendo presentear discentes com vivências tão ricas atreladas à saúde do trabalhador, apresentando-se como um diferencial na formação do profissional de saúde ao despertar nele um olhar diferenciado, cuidando para que seja capaz de desenvolver um olhar crítico-reflexivo sobre o ambiente de trabalho vivo que é o SUS ${ }^{(25)}$.

A construção de espaços colaborativos entre estudantes e profissionais é importante para que seja discutida a importância do pensar a saúde em todos os seus direcionamentos e públicos, pensando na troca de experiências e juntando o teórico ao prático ${ }^{(26)}$.

A promoção à saúde dos trabalhadores do SUS ainda é incipiente ${ }^{(27)}$, de modo que espaços como o gerado pela vivência relatada ajudam na promoção da saúde a partir de uma junção de conhecimentos e práticas, fortalecendo a busca por uma integração benéfica para os envolvidos nesse processo ${ }^{(28)}$.

Apesar da possibilidade da replicação da estratégia adotada para essa vivência em outros cenários, contextos e áreas de conhecimento, pode-se destacar como limitação do estudo que, por tratar-se de uma experiência vivencial, sua reprodução não garante o alcance das necessidades existentes em algum novo cenário de prática, principalmente pela necessidade de envolvimento dos profissionais que o compõem.

\section{CONCLUSÃO}

As ações executadas se mostraram de extrema relevância, despertando nos envolvidos a importância do autocuidado. Foram momentos ricos de troca de saber e de envolvimento dos profissionais e dos futuros profissionais de saúde, que puderam, nesses momentos, compreender a dinâmica dentro dos serviços de saúde e buscar 
alternativas para o desenvolvimento da promoção do cuidado dos profissionais de saúde que atuam na unidade analisada.

É essencial que a promoção à saúde do trabalhador do SUS esteja inclusa nas discussões durante a formação em saúde, que possa ser incorporada a vivências reais, para contribuir com o desenvolvimento desse espaço, que será seu futuro campo de atuação, ajudando a construir um espaço rico de saberes e aprendizados.

\section{CONFLITOS DE INTERESSE}

A descrição de experiência não possui relação que possa gerar quaisquer tipos de conflitos de interesses.

\section{CONTRIBUIÇÕES}

Isaac Newton Machado Bezerra, Vinicius Costa Maia Monteiro, Jânio Luiz do Nascimento, Larissa Oliveira Lima Macedo, Zacarias Ramalho Silvério, Aurélia de Oliveira Bento, Francisco Canindé dos Santos Silva e Jônia Cybele Santos Lima contribuíram com a elaboração e delineamento do estudo; a aquisição, análise e interpretação de dados; a redação e/ou revisão do manuscrito.

\section{REFERÊNCIAS}

1. Brasil. Constituição da República Federativa do Brasil. Brasília: Senado Federal; 1988.

2. Santana VS, Silva JM. Os 20 anos da saúde do trabalhador no Sistema Único de Saúde do Brasil: limites, avanços e desafios. In: Ministério da Saúde (BR). Secretaria de Vigilância em Saúde. Departamento de Análise de Situação de Saúde, organizadores. Saúde Brasil 2008: 20 anos de Sistema Único de Saúde no Brasil. Brasília: Ministério da Saúde; 2009. p. 175-204.

3. Brasil. Ministério da Saúde. Portaria GM n 3.252 de 22 de dezembro de 2009. Aprova as diretrizes para execução e financiamento das ações de Vigilância em Saúde pela União, Estados, Distrito Federal e Municípios e dá outras providências [Internet]. Diário Oficial da União; Brasília, 22 de dezembro de 2009 [acesso em 2018 Dez 20]. Disponível em: http://bvsms.saude.gov.br/bvs/saudelegis/gm/2009/ prt3252_22_12_2009.html

4. Brasil. Ministério da Saúde. Portaria GM no 1.378 de 09 de julho de 2013. Regulamenta as responsabilidades e define diretrizes para execução e financiamento das ações de Vigilância em Saúde pela União, Estados, Distrito Federal e Municípios, relativos ao Sistema Nacional de Vigilância em Saúde e Sistema Nacional de Vigilância Sanitária [Internet]. Diário Oficial da União; Brasília, 09 de julho de 2013 [acesso em 2018 Dez 20]. Disponível em: http://bvsms.saude.gov.br/bvs/saudelegis/gm/2013/prt1378_09_07_2013.html

5. Mendes JMR, Wünsch DS, Machado FSK, Martins J, Giongo CR. Saúde do trabalhador: desafios na efetivação do direito à saúde. Argumentum [Internet]. 2015 [acesso em 2019 Jan 08];7(2):194. Disponível em: http://periodicos.ufes.br/argumentum/article/view/10349

6. Ministério da Saúde (BR). Política Nacional de Promoção da Saúde. Brasília: Ministério da Saúde; 2006.

7. Carreiro GSP, Ferreira MO Filha, Lazarte R, Silva AO, Dias MD. O processo de adoecimento mental do trabalhador da Estratégia Saúde da Família. Rev Eletrônica Enferm [Internet]. 2013 [acesso em 2018 Dez 15];15(1):5-10. Disponível em: http://www.jyoungpharm.org/article/1051

8. Saborío ML, Hidalgo MLF. Síndrome de Burnout. Med Leg Costa Rica [Internet]. 2015 [acesso em 2019 Jun 05];32(1):119-24. Disponível em: http://www.scielo.sa.cr/scielo.php?script=sci_arttext\&pid=S140900152015000100014\&lng=en

9. Medeiros PA, Silva LC, Amarante IM, Cardoso VG, Mensch KM, et al. Condições de saúde entre profissionais da Atenção Básica em Saúde do Município de Santa Maria - RS. Rev Bras Ciênc Saúde. 2016;20(2):115-22.

10. Ministério da Saúde (BR). Protocolo da Mesa Nacional de Negociação Permanente do Sistema Único de Saúde - MNNP - SUS. Protocolo Nº08/2011 [Internet]. 2011 [acesso em 2018 Dez 20]. Disponível em: http://portalms.saude.gov.br/trabalho-educacao-e-qualificacao/gestao-e-regulacao-do-trabalho-em-saude/ mesa-nacional-do-sus/protocolos-e-resolucoes 
11. Moretto MLT, Jaen AC, Benute GRG, Ferrari S, Lucia MCS, Pollara W. "Cuidando de quem cuida": assistência psicológica ao trabalhador da saúde. Psicol Hosp [Internet]. 2013 [acesso em 2019 Jun 05];11(1):52-65. Disponível em: http://pepsic.bvsalud.org/scielo.php?script=sci_arttext\&pid=S1677-74092013000100004\&lng= pt\&nrm=iso

12. Munyewende PO, Rispel LC, Chirwa T. Positive practice environments influence job satisfaction of primary health care clinic nursing managers in two South African provinces. Hum Resour Health [Internet]. 2014 [acesso em 2019 Jun 05];12(27):1-14. Disponível em: http://www.ncbi.nlm.nih.gov/pmc/articles/PMC4024627

13. Shi L, Song K, Rane S, Sun X, Li H, Meng Q. Factors associated with job satisfaction by Chinese primary care providers. Prim Health Care Res Dev [Internet]. 2014 [acesso em 2019 Jun 05];15(1):46-57. Disponível em: http://www.ncbi.nlm.nih.gov/pubmed/23388523

14. Teles MA, Barbosa MR, Vargas AMD, Gomes VE, Ferreira EF, Martins AMBEBL, et al. Psychosocial work conditions and quality of life among primary health care employees: a cross sectional study. Health Qual Life Outcomes [Internet]. 2014 [acesso em 2019 Jun 05];72:1-12. Disponível em: http://www.ncbi.nlm.nih.gov/ pmc/articles/PMC4122097/pdf/1477-7525-12-72.pdf

15. Segura O. Burnout: concepts and implications affecting public health. Biomédica [Internet]. 2014 [acesso em 2019 Fev 08];34(4):535-45. Disponível em: http://www.scielo.org.co/scielo.php?script=sci_arttext\&pid=S012041572014000400006\&lng=en

16. Nunes MAP, Santana VR, Reis FP, Machado J Neto, Lima SO. A síndrome de Burnout em profissionais da Rede de Atenção Primária à Saúde de Aracaju, Brasil. Ciênc Saúde Colet [Internet]. 2015 [acesso em 2019 Fev 04];20(10):3011-20. Disponível em: http://www.scielo.br/scielo.php?script=sci_arttext\&pid=S1413$81232015001003011 \&$ Ing $=$ en

17. Martins LF, Laport TJ, Menezes VP, Medeiros PB, Ronzani TM. Esgotamento entre profissionais da Atenção Primária à Saúde. Ciênc Saúde Colet [Internet]. 2014 [acesso em 2019 Fev 04];19(12):4739-50. Disponível em: http://www.scielo.br/scielo.php?script=sci_arttext\&pid=S1413-81232014001204739\&lng=en

18. Lima AS, Farah BF, Bustamante-Teixeira MT. Análise da prevalência da síndrome de Burnout em profissionais da atenção primária em saúde. Trab Educ saúde [Internet]. 2018 [acesso em $2019 \mathrm{Fev}$ 05];16(1):283-304. Disponível em: http://www.scielo.br/scielo.php?script=sci_arttext\&pid=S1981-77462018000100283\&lng=pt\&nr $\mathrm{m}=$ iso

19. Ministério da Saúde (BR). Secretaria de Atenção à Saúde. Departamento de Atenção Básica. Política nacional de práticas integrativas e complementares no SUS: atitude de ampliação de acesso. $2^{\mathrm{a}}$ ed. Brasília: Ministério da Saúde; 2015.

20. Ministério da Saúde (BR). Glossário temático Práticas Integrativas e Complementares em Saúde [Internet]. Brasília: Ministério da Saúde; 2018 [acesso em 2018 Maio 31]. Disponível em: portalarquivos2.saude.gov.br/ images/pdf/2018/marco/12/glossario-tematico.pdf

21. Rodrigues NJF, Faria AA, Figueiredo MFS. Medicina complementar e alternativa: utilização pela comunidade de Montes Claros, Minas Gerais. Rev Assoc Med Bras [Internet]. 2009 [acesso em 2019 Fev 06];55(3):296-301. Disponível em: http://www.scielo.br/scielo.php?script=sci_arttext\&pid=S0104$42302009000300022 \&$ lng $=e n$

22. Gontijo MBA, Nunes MF. Integrative and complementary practices: knowledge and professional credibility of the public health service. Trab Educ Saúde [Internet]. 2017 [acesso em 2019 Fev 06];15(1):301-20. Disponível em: http://www.scielo.br/scielo.php?script=sci_arttext\&pid=S1981-77462017000100301\&lng=en\&n rm=iso

23. Sousa IMC, Bodstein RCA, Tesser CD, Santos FAS, Hortale VA. Integrative and complementary health practices: the supply and production of care in the Unified National Health System and in selected municipalities in Brazil. Cad Saúde Pública [Internet]. 2012 [acesso em 2019 Fev 06];28(11):2143-54. Disponível em: http://www.scielo.br/pdf/csp/v28n11/14.pdf

24. Telesi E Jr. Práticas integrativas e complementares em saúde, uma nova eficácia para o SUS. Estud Av [Internet]. 2016 [acesso em 2019 Fev 06];30(86):99-112. Disponível em: http://www.scielo.br/scielo. php?script=sci_arttext\&pid=S0103-40142016000100099\&lng=en\&nrm=iso 
25. Vendruscolo C, Prado ML, Kleba ME. Integração ensino-serviço no âmbito do Programa Nacional de Reorientação da Formação Profissional em Saúde. Ciênc Saúde Colet [Internet]. 2016 [acesso em 2019 Jun 12];21(9):2949-60. Disponível em: http://www.scielo.br/scielo.php?script=sci_arttext\&pid=S1413$81232016000902949 \&$ Ing=pt

26. Vendruscolo C, Trindade LL, Prado ML, Kleba ME. Repensando o modelo de Atenção em Saúde mediante a reorientação da formação. Rev Bras Enferm [Internet]. 2018 [acesso em 2019 Jun 12];71(Supl 4):1580-88. Disponível em: http://www.scielo.br/scielo.php?script=sci_arttext\&pid=S0034-71672018001001580\&lng=pt

27. Soratto J, Pires DEP, Trindade LL, Oliveira JSA, Forte ECN, Melo TP. Insatisfação no trabalho de profissionais da saúde na estratégia saúde da família. Texto \& Contexto Enferm [Internet]. 2017 [acesso em 2019 Jun 12];26(3):e2500016. Disponível em: http://www.scielo.br/scielo.php?script=sci_arttext\&pid=S010407072017000300325\&lng=en

28. Pizzinato A, Gustavo AS, Santos BRL, Ojeda BS, Ferreira E, Thiessen FVT, et al. A integração ensino-serviço como estratégia na formação profissional para o SUS. Rev Bras Educ Med [Internet]. 2012 [acesso em 2019 Jun 12];36(1 Supl 2):170-77. Disponível em: http://www.scielo.br/scielo.php?script=sci_arttext\&pid=S0100$55022012000300025 \&$ Ing=pt\&nrm=iso

\section{Endereço para correspondência:}

Isaac Newton Machado Bezerra

Universidade Federal do Rio Grande do Norte - UFRN

Avenida Senador Salgado Filho, 3000

Bairro: Candelária

CEP: 59064-741 - Natal - RN - Brasil

E-mail: isaac.ufrn30@gmail.com

Como citar: Bezerra INM, Monteiro VCM, Nascimento JL, Macedo LOL, Silvério ZR, Bento AO, et al. Práticas integrativas e complementares em saúde junto a profissionais da atenção primária. Rev Bras Promoç Saúde. 2019;32:9265. 